\title{
Highly Stereocontrolled Synthesis of the ABCD Ring Fragment of Ciguatoxin CTX3C
}

Tohru Oishi, ${ }^{1}$ Shin-ichiro Tanaka, Yoshihiro Ogasawara, Kenji Maeda, Hiroki Oguri, Masahiro Hirama*

Department of Chemistry, Graduate School of Science, Tohoku University, and CREST, Japan Science and Technology Corporation (JST), Sendai 980-8578, Japan

Fax +81 22 2176566; E-mail: hirama@ykbsc.chem.tohoku.ac.jp

Received 18 January 2001

This paper is dedicated to Professor Ryoji Noyori for his outstanding achievements in synthetic organic chemistry.

Abstract: A combination of asymmetric alkylation using $(1 R, 2 S)$ 1-amino-2-indanol derivative as a chiral auxiliary and the ring-closing metathesis reaction was shown to be an efficient method for synthesizing the $\mathrm{ABCD}$ ring fragment of ciguatoxin CTX3C.

Key words: ciguatoxin $\mathrm{CTX} 3 \mathrm{C}$, polycyclic ether, asymmetric alkylation, ring-closing metathesis, multiple asymmetric induction

Ciguatoxin CTX3C (1) is one of the causative toxins of ciguatera seafood poisoning prevalent in the tropics and subtropics. ${ }^{2}$ During the course of our synthetic studies directed toward ciguatoxins, ${ }^{3,4}$ we developed a highly convergent strategy for synthesizing polycyclic ethers based on a combination of alkylation and ring-closing metathesis (RCM) reactions, ${ }^{5}$ and have recently succeeded in synthesizing the ABCDE ring fragments of ciguatoxins. ${ }^{6}$ However, stereoselectivity of the alkylation step in the synthesis of the ABCDE fragment of $\mathbf{1}$ was low and the unnatural type diastereomer even predominated. Therefore, it became necessary to epimerize the epimer at a specific stage, lengthening the synthesis procedure that had only a moderate overall yield. ${ }^{6 a}$ In this paper, we describe the efficient synthesis of the ABCD ring fragment (2) of 1 through highly stereoselective alkylation and $\mathrm{RCM}$ reactions.
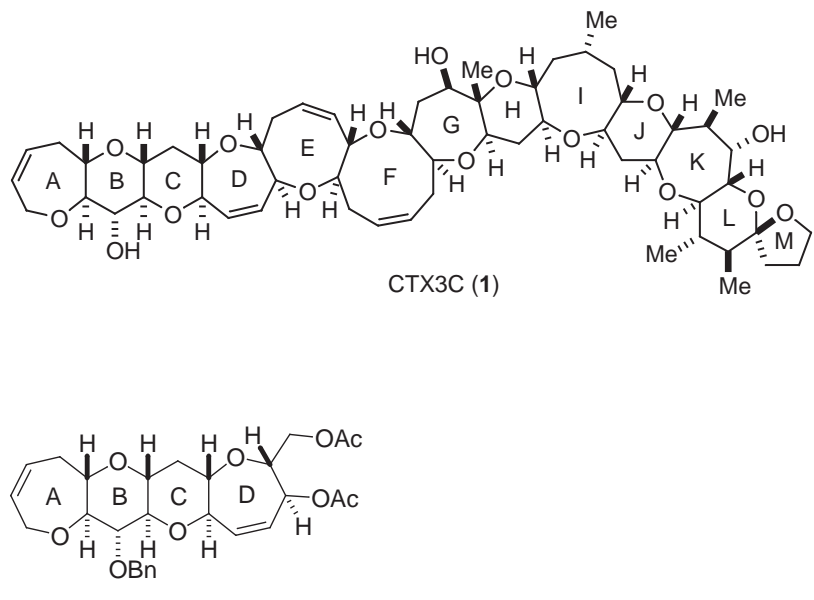

2
Our convergent synthesis of $\mathbf{2}$ began with the alkylation of $t$-butyl ester $4^{7}$ with iodide $\mathbf{3}^{6}$, corresponding to the AB ring of $\mathbf{1}$. As shown in the Table, chemical yield and diastereoselectivity were far from satisfactory (entries 1 and 2 ). The use of $N, N^{\prime}$-dimethylpropyleneurea (DMPU) as a co-solvent increased the yield but worsened selectivity. After numerous experiments, an amide $\mathbf{5}$ possessing an acetonide protected $(1 R, 2 S)$-1-amino-2-indanol as a chiral auxiliary ${ }^{8}$ was found to be suitable. The reaction using LDA afforded a 2:1 ratio in favor of a desired 7 (entry 3). The use of butyllithium as a base ${ }^{9}$ remarkably increased the 7:9 ratio to $10: 1$, but the yield was again low, irrespective of the presence or absence of hexamethylphosphoramide (HMPA) (entries 4 and 5). Finally, we found that addition of DMPU dramatically increased the chemical yield $(96 \%)$ without affecting the selectivity (10:1) (entry 6), while excess 5 was required to attain a high yield of 7 (entries 7 and 8). Thus, a multiple asymmetric induction improved the diastereoselectivity by a factor of $100 .^{10}$
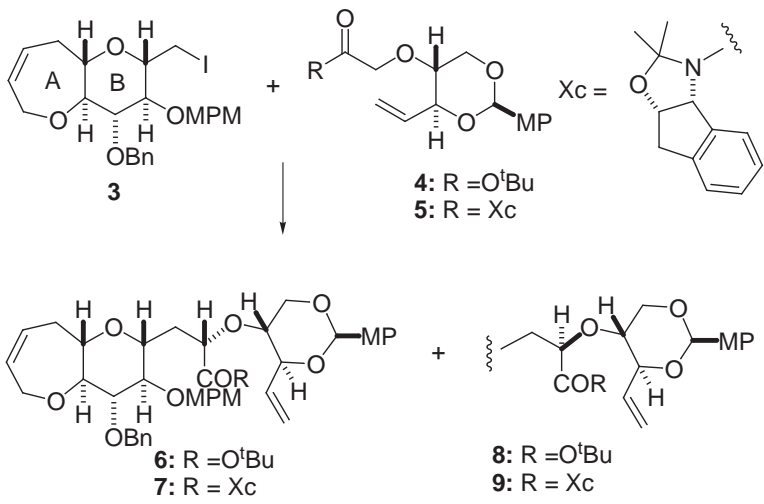

Scheme 1

Transformation of $\mathbf{7}$ to the ABCD ring fragment $\mathbf{2}$ is shown in Scheme 2. Selective removal of the $p$-methoxybenzylidene acetal of 7 using pyridinium $p$-toluenesulfonate (PPTS) in 1-propanol and the subsequent protection of the resulting 1,3-diol as bis- $t$-butyldiphenylsilyl (bis-TBPS) ether afforded 10 in $75 \%$ yield (2 steps). Removal of the MPM group of $\mathbf{1 0}$ gave alcohol 11, which was treated with camphorsulfonic acid (CSA) in toluene at $80{ }^{\circ} \mathrm{C}$ to yield lactone $12(60 \%)$. Although addition of 
Table Alkylation of $\mathbf{4}$ and $\mathbf{5}$ with Iodide $\mathbf{3}^{\text {a }}$

\begin{tabular}{cccccc}
\hline entry & base $^{\mathrm{b}}$ & solvent $^{\mathrm{c}}$ & $\mathbf{4}$ or $\mathbf{5}(\mathrm{eq})^{\mathrm{d}}$ & yield/\% & $\mathbf{6 ( 7 ) : 8 ( 9 )}$ \\
\hline 1 & LDA & THF-HMPA & $\mathbf{4}(2)$ & 23 & $1: 2$ \\
2 & LDA & THF-DMPU & $\mathbf{4}(2)$ & 43 & $1: 10$ \\
3 & LDA & THF & $\mathbf{5 ( 4 )}$ & 26 & $2: 1$ \\
4 & BuLi & THF & $\mathbf{5 ( 4 )}$ & 25 & $10: 1$ \\
5 & BuLi & THF-HMPA & $\mathbf{5 ( 4 )}$ & 26 & $10: 1$ \\
6 & BuLi & THF-DMPU & $\mathbf{5 ( 4 )}$ & 96 & $10: 1$ \\
7 & BuLi & THF-DMPU & $\mathbf{5 ( 2 )}$ & 73 & $10: 1$ \\
8 & BuLi & THF-DMPU & $\mathbf{5 ( 1 )}$ & 26 & $10: 1$ \\
\hline
\end{tabular}

${ }^{\mathrm{a} C}$ Carried out at $-78{ }^{\circ} \mathrm{C}$ to rt.

${ }^{\mathrm{b}}$ One equivalent of base was added to $\mathbf{4}$ or $\mathbf{5}$.

${ }^{\mathrm{c}}$ Ratio of THF-HMPA or THF-DMPU is $10: 1(\mathrm{v} / \mathrm{v})$.

${ }^{\mathrm{d}}$ Based on iodide 3.

${ }^{\mathrm{e}}$ Determined by $500 \mathrm{MHz}{ }^{1} \mathrm{H}$ NMR.

vinylmagnesium bromide resulted in a low yield of $\mathbf{1 3}$, vinyllithium generated from tributyl(vinyl)tin and methyllithium reacted with $\mathbf{1 2}$ and gave $\mathbf{1 3}$ in 98\% yield. Conversion of the hemiacetal $\mathbf{1 3}$ to the methylacetal $\mathbf{1 4}$ as a mixture of diastereomers in a 2:1 ratio and the subsequent reduction with triethylsilane in the presence of $\mathrm{BF}_{3} \cdot \mathrm{OEt}_{2}$ gave 15 . $^{31,3 \mathrm{~m}, 5,6}$ The RCM reaction ${ }^{3 \mathrm{i}-3 \mathrm{~m}, 5,11}$ of the diene 15 using Grubbs catalyst $(\mathbf{1 7})^{12}$ was too sluggish, presumably due to steric hindrance of the TBPS groups. ${ }^{6 a}$ The bis-TBPS ether 15 was then converted to the less hindered diacetate 16 in two steps (85\%). The RCM reaction of $\mathbf{1 6}$ proceeded smoothly to afford the ABCD ring fragment (2) of $\mathbf{1}$ in $74 \%$ yield. ${ }^{13}$ The stereochemistry of $\mathbf{2}$ was unambiguously determined by NOE experiments (Scheme 2).

In conclusion, a highly convergent, efficient and stereoselective synthesis of the ABCD ring fragment 2 of CTX3C (1) was achieved. The present asymmetric alkylation and ring-closing metathesis sequence will serve as a powerful tool for the synthesis of polycyclic ether toxins.

\section{References and Notes}

(1) Present address: Department of Chemistry, Graduate School of Science, Osaka University, Osaka 560-0043, Japan

(2) (a) Murata, M.; Legrand, A. M.; Ishibashi, Y.; Fukui, M.; Yasumoto, T. J. Am. Chem. Soc. 1990, 112, 4380; (b) Satake, M.; Murata, M.; Yasumoto, T. Tetrahedron Lett. 1993, 34, 1975; (c) Satake, M.; Morohashi, A.; Oguri, H.; Oishi, T.; Hirama, M.; Harada, N.; Yasumoto, T. J. Am. Chem. Soc. 1997, 119, 11325; (d) Satake, M.; Fukui, M.; Legrand, A. M.; Cruchet, P.; Yasumoto, T. Tetrahedron Lett. 1998, 39, 1197.

(3) (a) Suzuki, T.; Sato, O.; Hirama, M.; Yamamoto, Y.; Murata, M.; Yasumoto, T.; Harada, N. Tetrahedron Lett. 1991, 32 4505; (b) Oguri, H.; Hishiyama, S.; Oishi, T.; Hirama, M. Synlett 1995, 1252; (c) Oishi, T.; Shoji, M.; Maeda, K.; Kumahara, N.; Hirama, M. Synlett 1996, 1165; (d) Oguri, H.; Hishiyama, S.; Sato, O.; Oishi, T.; Hirama, M.; Murata, M.; Yasumoto, T.; Harada, N. Tetrahedron 1997, 53, 3057; (e) Oishi, T.; Shoji, M.; Kumahara, N.; Hirama, M. Chem. Lett. 1997, 845; (f) Oishi, T.; Maeda, K.; Hirama, M. Chem. Commun. 1997, 1289; (g) Oishi, T.; Maruyama, M.; Shoji, M.; Maeda, K.; Kumahara, N.; Tanaka, S.; Hirama, M. Tetrahedron 1999, 55, 7471; (h) Oguri, H.; Tanaka, S.; Hishiyama, S.; Oishi, T.; Hirama, M.; Tsumuraya, T.; Tomioka, Y.; Mizugaki, M. Synthesis 1999, 1431; (i) Oishi, T.; Nagumo, Y.; Hirama, M. Synlett 1997, 980; (j) Oguri, H.; Sasaki, S.; Oishi, T.; Hirama, M. Tetrahedron Lett. 1999, 40, 5405; (k) Oguri, H.; Tanaka, S.; Oishi, T.; Hirama, M. Tetrahedron Lett. 2000, 41, 975; (1) Oishi, T.; Nagumo, Y.; Shoji, M.; Le Brazidec, J.-Y.; Uehara, H.; Hirama, M. Chem. Commun. 1999, 2035; (m) Oishi, T.; Uehara, H.; Nagumo, Y.; Shoji, M.; Le Brazidec, J.-Y.; Kosaka, M.; Hirama, M. submitted for publication.

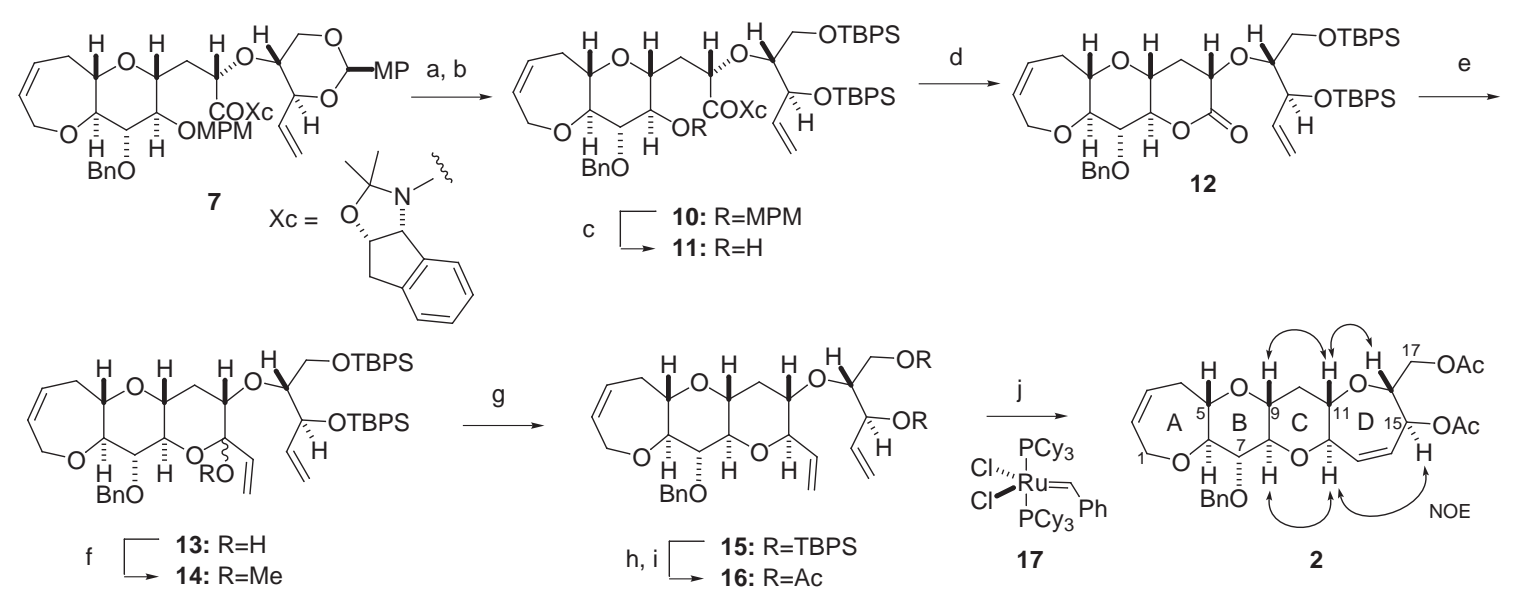

Scheme 2 Reagents and conditions. (a) PPTS, 1-propanol, rt, 5 h, 82\%; (b) TBPSCl, imidazole, DMF, $60{ }^{\circ} \mathrm{C}, 6$ h, $92 \%$; (c) $\mathrm{DDQ}^{\circ} \mathrm{CH}_{2} \mathrm{Cl}_{2}$, $\mathrm{H}_{2} \mathrm{O}$, rt, $1 \mathrm{~h}, 75 \%$; (d) CSA, toluene, $80{ }^{\circ} \mathrm{C}, 1 \mathrm{~d}, 60 \%$; (e) tributyl(vinyl)tin, $\mathrm{MeLi}, \mathrm{THF},-78{ }^{\circ} \mathrm{C}, 30 \mathrm{~min}, 98 \%$; (f) $\mathrm{HC}(\mathrm{OMe})_{3}, \mathrm{p}-\mathrm{TsOH} \bullet \mathrm{H}_{2} \mathrm{O}$, $\mathrm{CH}_{2} \mathrm{Cl}_{2}, \mathrm{rt}, 12 \mathrm{~h}, 70 \%$; (g) $\mathrm{Et}_{3} \mathrm{SiH}, \mathrm{BF}_{3} \bullet \mathrm{OEt}_{2}, \mathrm{CH}_{2} \mathrm{Cl}_{2},-30{ }^{\circ} \mathrm{C}, 2 \mathrm{~h}, 61 \%$; (h) TBAF, THF, rt, 4 h; (i) Ac $2 \mathrm{O}, \mathrm{Py}, \mathrm{DMAP}, \mathrm{rt}, 4 \mathrm{~h}, 85 \%$ (2 steps); (j) $17, \mathrm{CH}_{2} \mathrm{Cl}_{2}$, reflux, $12 \mathrm{~h}, 74 \%$. 
(4) For recent synthetic studies of other groups, see: (a) Oka, T.; Murai, A. Tetrahedron 1998, 54, 1; (b) Oka, T.; Fujiwara, K.; Murai, A. Tetrahedron 1998, 54, 21; (c) Inoue, M.; Sasaki, M.; Tachibana, K. Angew. Chem., Int. Ed. Engl. 1998, 37, 965;

(d) Sasaki, M.; Noguchi, T.; Tachibana, K. Tetrahedron Lett. 1999, 40, 1337; (e) Hosokawa, S.; Isobe, M. J. Org. Chem. 1999, 64, 37; (f) Saeeng, R.; Isobe, M. Tetrahedron Lett. 1999, 40, 1911; (g) Sasaki, M.; Fuwa, H.; Ishikawa, M.; Tachibana, K. Org. Lett. 1999, 1, 1075.

(5) Oishi, T.; Nagumo, Y.; Hirama, M. Chem. Commun. 1998, 1041.

(6) (a) Maruyama, M.; Maeda, K.; Oishi, T.; Oguri, H.; Hirama, M. Heterocycles 2001, 54, 93; (b) Maeda, K.; Oishi, T.; Oguri, H.; Hirama, M. Chem. Commun. 1999, 1063.

(7) Synthesized from D-glucose: (i) $p$-methoxybenzaldehyde dimethylacetal, $p$-toluenesulfonic acid, DMF, $45 \%$; (ii) $\mathrm{NaIO}_{4}, \mathrm{MeOH}$, pH6 acetate buffer; (iii) $\mathrm{Ph}_{3} \mathrm{PCH}_{3} \mathrm{Br}, t$-BuOK, THF, $58 \%$ (2 steps); (iv) $t$-butyl bromoacetate, $\mathrm{NaH}$, THF, DMF, $84 \%$.

(8) (a) Askin, D.; Wallace, M. A.; Vacca, J. P.; Reamer, R. A.; Volante, R. P.; Shinkai, I. J. Org. Chem. 1992, 57, 2771; (b) Askin, D.; Eng, K. K.; Rossen, K.; Purick, R. M.; Wells, K. M.; Volante, R. P.; Reider, P. J. Tetrahedron Lett. 1994, 35, 673.

(9) $t$-Butyllithium is also usable and gave a very similar result.

(10) Masamune, S.; Choy, W.; Petersen, J. S.; Sita, L. R. Angew. Chem., Int. Ed. Engl. 1985, 24, 1.

(11) For the synthetic studies of cyclic polyethers using RCM reported by other groups, see: (a) Nicolaou, K. C.; Postema, M. H. D.; Claiborne, C. F. J. Am. Chem. Soc. 1996, 118, 1565; (b) Nicolaou, K. C.; Postema, M. H. D.; Yue, E. W.; Nadin, A. J. Am. Chem. Soc. 1996, 118, 10335; (c) Clark, J. S.; Kettle, J. G. Tetrahedron Lett. 1997, 38, 123; (d) Clark, J. S.; Kettle, J. G. Tetrahedron Lett. 1997, 38, 127; (e) Rainier, J. D.; Allwein, S. P. J. Org. Chem. 1998, 63, 5310; (f) Clark, J. S.; Hamelin, O.; Hufton, R. Tetrahedron Lett. 1998, 39, 8321; (g) Clark, J. S.; Kettle, J. G. Tetrahedron 1999, 55, 8231; (h) Eriksson, L.;
Guy, S.; Perlmutter, P. J. Org. Chem. 1999, 64, 8396; (i) Crimmins, M. T.; Choy, A. J. Am. Chem. Soc. 1999, 121, 5653; (j) Crimmins, M. T.; Emmitte, K. A. Synthesis 2000, 899. Recent reviews on ring-closing metathesis reactions, see: (k) Armstrong, S. K. J. Chem. Soc., Perkin Trans. 1 1998, 371; (l) Grubbs, R. H.; Chang, S. Tetrahedron 1998, 54, 4413; (m) Schuster, M.; Blechert, S. Angew. Chem., Int. Ed. Engl. 1997, 36, 2036.

(12) (a) Fu, G. C.; Nguyen, S. T.; Grubbs, R. H. J. Am. Chem. Soc. 1993, 115, 9856; (b) Miller, S. J.; Kim, S.-H.; Chen, Z.-R.; Grubbs, R. H. J. Am. Chem. Soc. 1995, 117, 2108; (c) Miller, S. J.; Grubbs, R. H. J. Am. Chem. Soc. 1995, 117, 5855.

(13) Physical data of 2: $[\alpha]_{\mathrm{D}}^{30}-6.33\left(c 0.42, \mathrm{CHCl}_{3}\right)$; IR (film) $v$ $3027,2932,1744,1453,1369,1241,1107,753,697 \mathrm{~cm}^{-1} ;{ }^{1} \mathrm{H}$

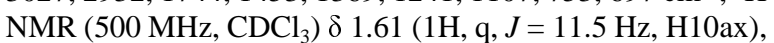
$2.07(3 \mathrm{H}, \mathrm{s}, \mathrm{Ac}), 2.08(3 \mathrm{H}, \mathrm{s}, \mathrm{Ac}), 2.29-2.38(2 \mathrm{H}, \mathrm{m}, \mathrm{H} 4$, H10eq), 2.63 (1H, ddd, $J=16.5,8.0,4.0 \mathrm{~Hz}, \mathrm{H} 4), 3.09-3.16$ $(2 \mathrm{H}, \mathrm{m}, \mathrm{H} 8, \mathrm{H} 9), 3.27(1 \mathrm{H}, \mathrm{td}, J=9.5,4.0 \mathrm{~Hz}, \mathrm{H} 5), 3.34(1 \mathrm{H}$, $\mathrm{t}, J=9.5 \mathrm{~Hz}, \mathrm{H} 6), 3.36(1 \mathrm{H}, \mathrm{ddd}, J=11.5,9.5,5.0 \mathrm{~Hz}, \mathrm{H} 11)$, $3.47(1 \mathrm{H}, \mathrm{t}, J=9.5 \mathrm{~Hz}, \mathrm{H} 7), 3.79(1 \mathrm{H}$, ddd, $J=9.5,6.0,2.5$ $\mathrm{Hz}, \mathrm{H} 16), 3.94(1 \mathrm{H}, \mathrm{dd}, J=9.5,2.0 \mathrm{~Hz}, \mathrm{H} 12), 4.01(1 \mathrm{H}, \mathrm{dq}$, $J=15.0,3.0 \mathrm{~Hz}, \mathrm{H} 1), 4.04(1 \mathrm{H}, \mathrm{dd}, J=12.0,2.5 \mathrm{~Hz}, \mathrm{H} 17)$, 4.09 (1H, dd, $J=12.0,6.0 \mathrm{~Hz}, \mathrm{H} 17), 4.29(1 \mathrm{H}, \mathrm{dd}, J=15.0$, $6.0 \mathrm{~Hz}, \mathrm{H} 1), 4.83\left(1 \mathrm{H}, \mathrm{d}, J=12.0 \mathrm{~Hz}, \mathrm{OCH}_{2} \mathrm{Ph}\right), 4.88(1 \mathrm{H}, \mathrm{d}$, $\left.J=12.0 \mathrm{~Hz}, \mathrm{OCH}_{2} \mathrm{Ph}\right), 5.44(1 \mathrm{H}, \mathrm{dq}, J=9.5,2.0 \mathrm{~Hz}, \mathrm{H} 15)$, $5.52(1 \mathrm{H}, \mathrm{dt}, J=11.5,2.0 \mathrm{~Hz}, \mathrm{H} 14), 5.74(1 \mathrm{H}, \mathrm{dt}, J=11.5,2.0$ $\mathrm{Hz}, \mathrm{H} 13), 5.77(1 \mathrm{H}$, ddt, $J=12.0,8.0,3.0 \mathrm{~Hz}, \mathrm{H} 3), 5.86(1 \mathrm{H}$, ddt, $J=12.0,6.0,3.0 \mathrm{~Hz}, \mathrm{H} 2), 7.24-7.29(1 \mathrm{H}, \mathrm{m}), 7.33(2 \mathrm{H}, \mathrm{t}$, $J=7.5 \mathrm{~Hz}), 7.39(2 \mathrm{H}, \mathrm{d}, J=7.5 \mathrm{~Hz}) ;{ }^{13} \mathrm{C}$ NMR $(125 \mathrm{MHz}$, $\left.\mathrm{CDCl}_{3}\right) \delta 20.82,20.96,34.59,36.60,64.25,68.40,71.34$, 73.09, 75.15, 76.87, 79.06, 80.05, 80.41, 80.95, 82.06, 87.33, $126.65,127.43,127.71,128.19,129.97,131.33,132.39$, 139.12, 169.60, 170.82; MALDI-TOF MS calcd for $\mathrm{C}_{28} \mathrm{H}_{34} \mathrm{O}_{9}$ $(\mathrm{M}+\mathrm{Na})^{+}$537.21, found 537.13.

Article Identifier:

1437-2096,E;2001,0,SI,0952,0954,ftx,en;Y02101ST.pdf 